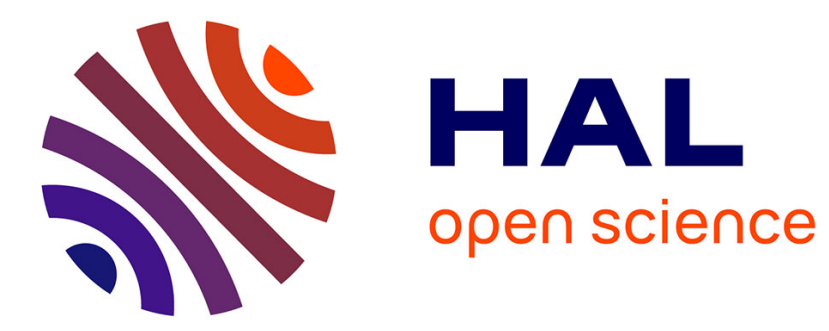

\title{
Education et finances publiques en Espagne de 1850 à 1965
}

Claude Diebolt

\section{To cite this version:}

Claude Diebolt. Education et finances publiques en Espagne de 1850 à 1965 . Revue d'économie financière, 2020, 2 (138), pp.297-300. 10.3917/ecofi.138.0297 . hal-02929539

\section{HAL Id: hal-02929539 \\ https://hal.science/hal-02929539}

Submitted on 3 Sep 2020

HAL is a multi-disciplinary open access archive for the deposit and dissemination of scientific research documents, whether they are published or not. The documents may come from teaching and research institutions in France or abroad, or from public or private research centers.
L'archive ouverte pluridisciplinaire HAL, est destinée au dépôt et à la diffusion de documents scientifiques de niveau recherche, publiés ou non, émanant des établissements d'enseignement et de recherche français ou étrangers, des laboratoires publics ou privés. 


\section{Education et finances publiques en Espagne de 1850 à 1965}

Claude Diebolt

Directeur de Recherche au CNRS cdiebolt@unistra.fr

Tout au long du 19ème siècle et jusqu'à la guerre civile de 1936 à 1939, le système éducatif espagnol est étroitement contrôlé, d'abord par l'Eglise, puis par l'Etat, et dirigé par la bourgeoisie toute puissante, remplacée à la veille de la Seconde Guerre mondiale par le triomphe du franquisme. Dans ces conditions, comment le système éducatif espagnol est-il parvenu, depuis la constitution de 1812, à sortir d'une inertie commandée par l'existence de sous-systèmes cloisonnés à fonctionnalité et à idéologie différentes ? La réponse à cette question se trouve, semble-t-il, dans la diversité des chocs et des défis que le système éducatif a reçu de l'extérieur. Le premier défi vient de l'accélération de l'évolution vers une économie de plus en plus technico-économique au sein de laquelle les problèmes scolaires ne peuvent plus se poser dans des termes empruntés au passé. Une société en croissance détermine indubitablement une demande sociale d'éducation élevée. Corrélativement, les planificateurs de l'éducation découvrent que l'efficacité de l'enseignement constitue l'un des moteurs de l'expansion. Celui de la croissance économique n'est pas le moindre car il détermine la pérennité et l'avenir de la société capitaliste. Aussi, on comprend aisément pourquoi le système éducatif espagnol adopte, historiquement, devant les conditions nouvelles qui lui sont faites, une philosophie nouvelle, ordonnant de manière plus originale et plus efficace peut-être, les temps de l'information, de la mise en ordre, de l'assimilation et du contrôle.

Au début du 19ème siècle, la faible densité de population constitue l'un des facteurs majeurs du retard socio-économique de l'Espagne. Un second facteur réside dans la remise en ordre des finances publiques et de la monnaie. Entre 1801 et 1814, les dépenses de l'Etat se trouvent dans une situation catastrophique. Entre 1814 et 1840 , la situation politique et administrative n'évolue guère. Figée dans un refus total des innovations, elle se caractérise par le passage d'un autoritarisme inefficace à la paralysie de la guerre civile. Qui plus est, la situation se complexifie avec une crise agraire qui ne s'achève qu'au courant des années 1830 . D'une manière plus générale, l'Espagne du début du 19ème siècle est face à une impossible industrialisation, avec certes des entreprises, mais pas d'industrie. En fait, il faut attendre les années 1850, pour enregistrer une véritable rupture. A partir du milieu du 19ème siècle, l'Espagne se dote de budgets réguliers et publiés. Ils représentent le fondement de la comptabilité publique d'une part, et celui de la stabilité politique et économique d'autre part. Cela étant, comparée à la Grande-Bretagne, à la France et à l'Allemagne, le pays demeure dans une situation d'isolement économique. Pourtant, à partir des années 1850, I'Espagne bénéficie de la conjonction de trois facteurs favorables à son développement socio-économique. Il s'agit de la conjoncture internationale à tendance expansionniste, de l'investissement étranger et de la stabilité politique. Cela dit, la crise de 1865-1866 marque un coup d'arrêt à cet élan de prospérité. La faillite des chemins de fer ébranle le système financier et l'Espagne enregistre sa première crise 
capitaliste qui connaît son apogée avec la guerre franco-allemande de 1870-1871. La dépression économique qui s'ensuit en 1873 n'est surmontée qu'en 1878. Mais, dès 1881 apparaît une autre crise internationale qui prolonge la stagnation économique et qui ne cesse qu'avec le tournant du siècle. Néanmoins, la phase de difficultés économiques des années 1873-1896 accélère les transformations dans les structures de production et d'échange. La réduction du prix des biens d'équipement par exemple, associés à de meilleurs gains de productivité, constitue un facteur favorable pour la croissance économique. En effet, au plan international, les développements de la sidérurgie, de la chimie et de l'électricité vont, d'une part favoriser l'exploitation des minerais disponibles, et d'autre part accompagner de profonds changements sociaux (essor de l'urbanisation, concentration ouvrière, etc...). En même temps, les figures 1 et 2 , ci-dessous, témoignent de la relative négligence de l'Etat espagnol pour le développement scolaire. En fait, mis à part le service de la dette, l'armée et les dépenses courantes d'administration absorbent l'essentiel des disponibilités publiques et, partant, une part importante de la richesse nationale. Ce n'est qu'après la première guerre mondiale, que le développement des dépenses en faveur de l'instruction publique permet d'entrevoir l'intégration progressive de l'économie espagnole au sein d'une Europe occidentale en plein essor. Pour autant, au plan économique comme pour l'éducation, les années 1920 et le début des années 1930 représentent la continuation des tendances de croissance apparues à la fin du 19ème siècle avec, en point d'orgue, la cassure économique et sociale liée à la guerre civile de 1936. Ce faisant, le développement de l'éducation, et plus particulièrement le phénomène d'éducation de masse, amorcé après 1945, est en décalage de deux décennies par rapport à des pays du centre de l'Europe comme la France ou l'Allemagne. Pour autant, à partir de la fin des années 1960 et le début des années 1970, l'Espagne passe progressivement d'un statut de nation économique retardée à une puissance économique majeure.

Figure 1

Les dépenses de l'Etat en matière d'éducation rapportées aux dépenses totales de l'Etat

Espagne, 1850-1965

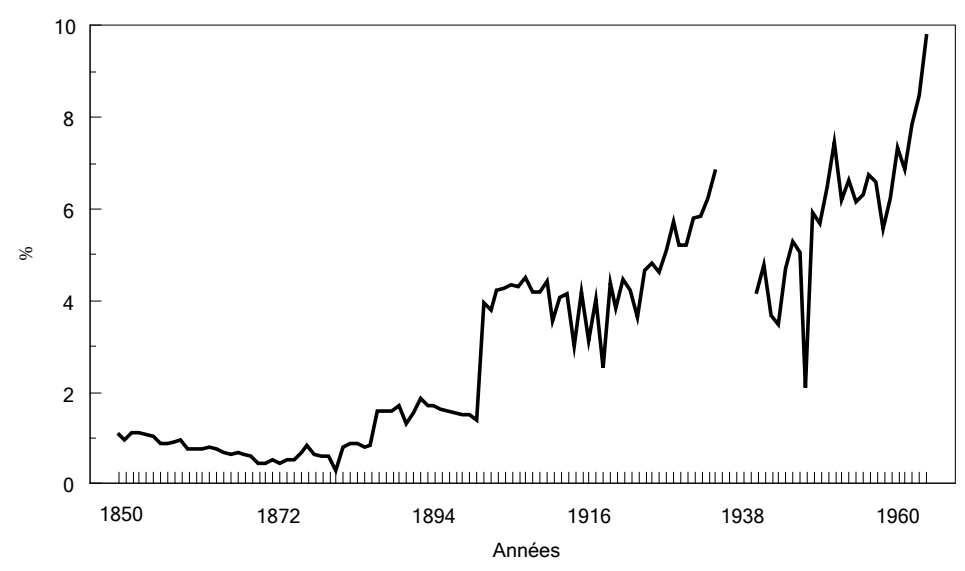

Source : Diebolt (2000) 
Figure 2

Les dépenses de l'Etat en matière d'éducation rapportées au revenu national aux coûts des facteurs

Espagne, 1850-1965

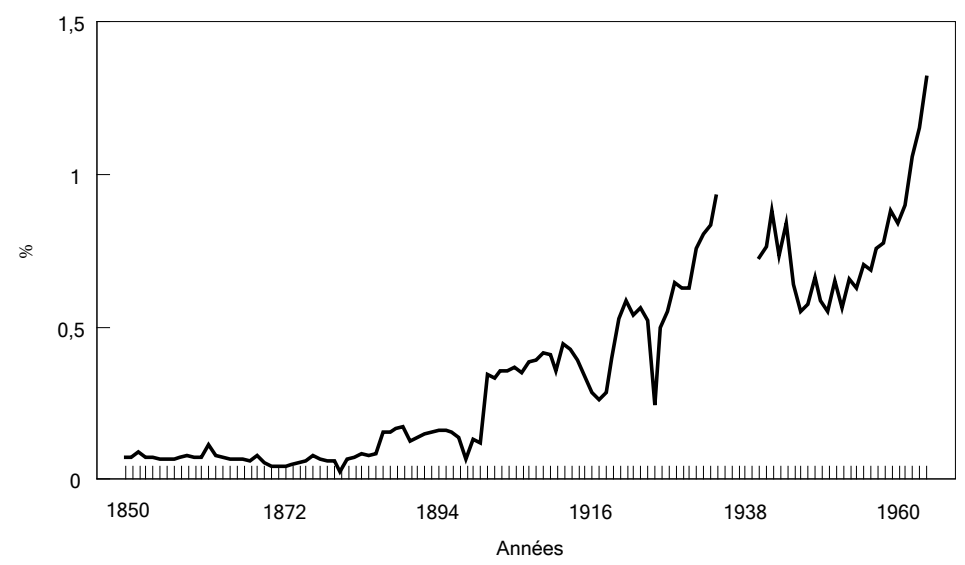

Source : Diebolt (2000)

Références :

Carreras, A. (édit.) : Estadisticas historicas de España siglos XIX-XX, Fundación Banco Exterior, Madrid, 1989.

Comin, F. : Hacienda y economía en la España contemporánea (1800-1936). Vol. I : El afianzamiento de la hacienda liberal (1800-1874), Vol. II. : La hacienda transicional (1875-1935), Ministerio de Economía y Hacienda, Madrid, 1988.

Diebolt, C.: Dépenses d'éducation et cycles économiques en Espagne aux XIXe et XXe siècles, L'Harmattan, Collection « Logiques Economiques », Paris, 2000.

Intervención General de la Administración del Estado (édit.) : Estadística de los presupuestos generales del estado y de los resultados que ha ofrecido su liquidación. Años 1850 á 1890-91, Fábrica Nacional de Moneda y Timbre, Madrid, 1891.

Ministerio de Hacienda (édit.) : Datos basicos para la historia financiera de España (1850-1975), 2 vol., Fábrica Nacional de Moneda y Timbre, Madrid, 1976.

Ministerio de Hacienda (édit.) : Estadisticas de la cuentas generales del estado. Años 1908 á 1923/1924, Fábrica Nacional de Moneda y Timbre, Madrid, 1979.

Núñez, C.E. : "El gasto público en educatión entre 1862 y 1935", in : Hacienda Pública Española, Monografias, $n^{\circ} 1,1991$, pp. 121-146. 